\title{
Examining the Presentation of Information in Augmented Reality Headsets for Situational Awareness
}

\author{
Julia Woodward \\ Department of CISE \\ University of Florida \\ Gainesville, Florida, USA \\ julia.woodward@ufl.edu
}

\author{
Jesse Smith \\ Department of CISE \\ University of Florida \\ Gainesville, Florida, USA \\ jd.smith@ufl.edu
}

\author{
Isaac Wang \\ Department of CISE \\ University of Florida \\ Gainesville, Florida, USA \\ wangi@ufl.edu
}

\author{
Sofia Cuenca \\ Department of Computer Systems \\ Farmingdale State College \\ Farmingdale, New York, USA \\ cuens@farmingdale.edu
}

\author{
Jaime Ruiz \\ Department of CISE \\ University of Florida \\ Gainesville, Florida, USA \\ jaime.ruiz@ufl.edu
}

\begin{abstract}
Augmented Reality (AR) headsets are being employed in industrial settings (e.g., the oil industry); however, there has been little work on how information should be presented in these headsets, especially in the context of situational awareness. We present a study examining three different presentation styles (Display, Environment, Mixed Environment) for textual secondary information in AR headsets. We found that the Display and Environment presentation styles assisted in perception and comprehension. Our work contributes a first step to understanding how to design visual information in $A R$ headsets to support situational awareness.
\end{abstract}

\section{CCS CONCEPTS}

- Human-centered computing $\rightarrow$ Mixed / augmented reality

\section{KEYWORDS}

Augmented reality, situational awareness

\section{ACM Reference format:}

Julia Woodward, Jesse Smith, Isaac Wang, Sofia Cuenca, and Jaime Ruiz. 2020. Examining the Presentation of Information in Augmented Reality Headsets for Situational Awareness. In Proceedings of the 2020 International Conference on Advanced Visual Interfaces (AVI'20). ACM, New York, NY, USA, 5 pages. https://doi.org/10.1145/3399715.3399846

Permission to make digital or hard copies of part or all of this work for personal or classroom use is granted without fee provided that copies are not made or distributed for profit or commercial advantage and that copies bear this notice and the full citation on the first page. Copyrights for components of this work owned by others than ACM must be honored. Abstracting with credit is permitted. To copy otherwise, or republish, to post on servers or to redistribute to lists, requires prior specific permission and/or a fee. Request permissions from Permissions@acm.org.

AVI '20, September 28-October 2, 2020, Salerno, Italy

(c) 2020 Association for Computing Machinery.

ACM ISBN 978-1-4503-7535-1/20/09...\$15.00

https://doi.org/10.1145/3399715.3399846

\section{Introduction}

Augmented reality (AR) headsets allow a user to see and interact with virtual objects projected onto a view of the real world, and are beginning to enter the consumer and industrial markets $[18,34]$. However, there has been little prior work on how information should be presented in AR headsets, especially in the context of situational awareness. Situational awareness is defined as "the detection of elements in the environment within a volume of space and time (level 1), the comprehension of their meaning (level 2), and the projection of their status in the near future (level 3)" [6,7]. Poor situational awareness has caused aircraft crashes [29] and errors in anesthesia [28].

$\mathrm{AR}$ has the potential to increase situational awareness through providing a secondary channel of information that can be overlaid over the real world. Prior work has examined applying AR for situational awareness in different contexts, such as the military [10]. However, there has been conflicting results, for instance higher and lower situational awareness [2,23,25]. Therefore, applying AR to a task may not improve situational awareness. In addition, previous studies have mainly focused on the applicability of $\mathrm{AR}$ and how it compares to traditional methods instead of information design (e.g., [14,22,23]). Since AR headsets have the potential to improve situational awareness [14], it is important to study how visual information should be presented to maximize awareness while minimizing distractions.

Visual information, in the context of awareness, can be split into two categories: central or critical (e.g., hazard warnings) and peripheral or secondary (e.g., nonessential information) [4]. While critical information should always be visually salient [8,9], secondary information does not have this constraint; allowing for larger quantities of detailed information (e.g., item descriptions) and increasing integration opportunities with the environment. Therefore, we investigated the presentation of secondary information in AR headsets to assist in levels 1 and 2 of situational awareness (perception and comprehension). We examined three different textual presentation styles: locked to 
the display view (Display), located in the environment (Environment), and a mix of both (Mixed Environment). We studied text because it is common and necessary in AR headset applications to effectively communicate information [14,21,23].

In our study, participants had to complete multiplication problems while monitoring the textual secondary information in an AR headset. We found that the Display and Environment presentation styles supported the recall of secondary information, when compared to the Mixed Environment style. We contribute a new understanding of how different presentation styles for textual secondary information in AR headsets support perception and comprehension. Our work is a first step in investigating the design of visual information in AR headsets to increase users' situational awareness.

\section{Background and Related Work}

Prior work has examined utilizing AR for situational awareness in safety critical domains [14,16,22,25,33]. Ruano et al. [25] created an AR system for the flight of Unmanned Aerial Vehicles (UAVs). The AR system overlaid flight mission data (e.g., route orientation) onto a live video stream on a computer screen, instead of having two separate screens as in previous UAV systems. The AR system was found to improve the situational awareness of the UAV operators. Park et al. [22] designed an AR system to increase driving situational awareness. The system was overlaid on the car windshield and would provide warning information (e.g., distance of another vehicle). While these prior studies have investigated utilizing AR for situational awareness, they only examined overlaying graphical elements onto current display screens (e.g., car windshields, computers), not using AR headsets. AR headsets offer more freedom, immersion, and contextual integration with the environment.

Liu et al. [14] investigated if an AR headset would aid anesthesiologists in monitoring patient information. In the study, 12 anesthesiologists provided anesthesia in a simulated environment. The anesthesiologists using the AR headset detected patient events faster. Zhu et al. [32] created AR-Mentor, a wearable AR mentoring system to assist in maintenance for complex machinery. AR-Mentor provides guidance through voice instruction and visual elements in an AR headset (e.g., 3D graphic animations). The authors conducted preliminary training tests with novice users and found that it demonstrated promising effectiveness. Both of the studies above show the potential of using AR headsets for situational awareness. However, the two studies did not investigate how the information should be presented in the headsets to aid in situational awareness.

\subsection{Readability of Information in AR}

Prior work has examined the readability of information in AR [1,12,21,27]. Rzayev et al. [27] examined how text should be displayed for reading in an AR headset while the user is walking vs. sitting. They compared three text positions (top-right, center, and bottom-center) and two presentation types, line-by-line scrolling and Rapid Serial Visual Presentation (RSVP). RSVP presents text word-by-word in a fixed location. Presenting the text in the top-right increased cognitive workload and reduced text comprehension. RSVP had higher comprehension during sitting, while line-by-line scrolling had higher comprehension during walking. Debernardis et al. [5] examined how text design affected readability in both optical and video see-through AR headsets. Participants were faster in readability with the optical see-through headset. The authors recommended using white text with a blue billboard (i.e., background). However, prior work has also recommended transparent backgrounds [1]. Albarelli et al. [1] analyzed the difference between transparent and opaque overlays in an AR headset. During the study, the participants stocked items in a test grocery store while product information was shown in the headset. The participants preferred a central display of information with a transparent background for readability. These prior studies examined the readability of text related to the main task in $\mathrm{AR}$, but not in terms of situational awareness. We focused on analyzing different presentation styles for textual secondary information in AR headsets.

\section{Method}

In our study, participants completed multiplication problems on a Wacom Cintiq Companion Hybrid tablet [30] while viewing the textual secondary information in a Meta 2 AR headset [18]. Both the AR and multiplication applications were created using Unity. We conducted the study in a room with consistent lighting, and the study took approximately 60 minutes. Participants either received extra credit for a course they were enrolled in or voluntarily participated without compensation. Our protocol was approved by our Institutional Review Board.

At the start of the study, participants filled out a demographic questionnaire, and then completed multiplication problems on the tablet for 5 minutes without wearing the AR headset. These problems were practice and not used in any analysis. After the practice, participants then put on the AR headset and began the main part of the study. In total, there were three different study blocks ( 5 minutes each), one block for each presentation style (Display, Environment, Mixed Environment). The participants would complete the block for a presentation style and then complete a NASA TLX survey [11] for that style. The NASA TLX Survey was used to determine the participants' perceived cognitive workload. Also, we asked the participants to recall the last textual information presented in the headset. We did not explain that we would ask for the last textual information presented in the headset, which allowed us to examine if there was a difference in perceptibility and comprehension. After the survey, the participants would then complete the next block with a different presentation style, and so forth. The order of the presentation styles was counterbalanced across participants.

\subsection{Participants}

The participants included 33 adults $(\mathrm{M}=21.55$ years, $\mathrm{SD}=3.55)$. Twelve participants were female and one participant identified as non-binary; two participants were left-handed. We excluded three participants: one due to equipment failure, and two due to self-reported peripheral vision loss. Therefore, we had a total of 

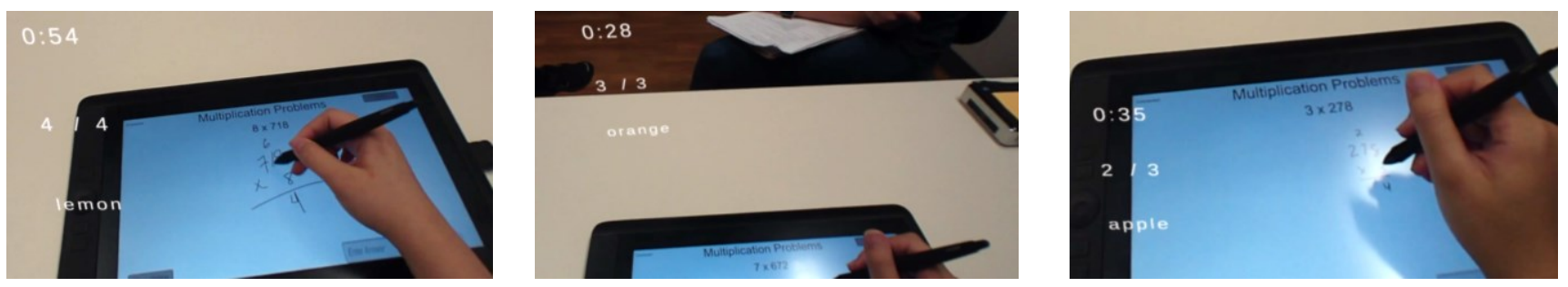

Figure 1. Presentation styles in AR headset: Display (left), Environment (middle), and Mixed Environment (right).

30 participants for analysis $(M=21.63$ years, $S D=3.69)$. All 30 participants had normal or corrected-to-normal vision.

\subsection{Design}

Each participant viewed the secondary textual information in three different presentation styles: locked to the display view (Display), located in the environment (Environment), and a mix of both (Mixed Environment). For the Display presentation style, the textual information was locked to the left-hand side of the field-of-view. We placed the information on the left-side due to the quantity of information (i.e., it would obstruct the participant's view in the center), and because prior work has found lower detection accuracy for the right-side [17]. The text height was $5 \mathrm{~mm}$ and white, which is aligned with Meta AR design recommendations [18]. The participants could always see the information in the headset (Figure 1a). In the Environment presentation style, the text appeared $500 \mathrm{~mm}$ away from the participant with a height of $10 \mathrm{~mm}$, which is consistent with design recommendations [19]. The text was fixed in the environment to the left of the participant, in order to be consistent with the other presentation styles (Figure 1b). In Figure $1 \mathrm{~b}$ the participant is looking at the text in the headset and therefore able to clearly see it. However, if the participant looked straight down at the tablet or turned their head all the way to the right or left, they would not be able to see the text since it was in a fixed location in the environment. The Mixed Environment presentation style was a mix of both the previous styles. The text was always present in the field-of-view (similar to Display) but appeared $500 \mathrm{~mm}$ away from the participant with a height of $10 \mathrm{~mm}$ (similar to Environment). As with the Display style, the participant was always able to see the information in the headset. However, having the text farther away from the participant made the text appear less spread out and further into the participant's central vision (Figure 1c).

3.2.1 AR Secondary Textual Information. The secondary information included the participant's average math problem completion time, math accuracy, and a random word (Figure 1). We used Liberation Sans font since it is recommended for readability [24]. There was $75 \mathrm{~mm}$ between each textual element. The average math completion time was the average time it took the participant to solve the problems, presented in minutes and seconds. The math accuracy was a ratio of the number of correctly answered problems by the number of completed problems. The average time and accuracy were calculated in real-time and would update in the headset after each problem.
Lastly, for the random word, it would randomly cycle between: "banana", "apple", "orange", and "lemon". We used the random word as a proxy for information that might not be directly related to the main task but still necessary for maintaining situational awareness. In addition, it allowed us to further examine the perceptibility of the presentation styles. The current word would remain visible in the headset for a random time (between 20-40 seconds) before switching to the next word.

3.2.2 Math Application Design. We used math as the main task because it utilizes working memory [15]. Working memory is a part of short-term memory that is concerned with perceptual processing tasks [3]. Having participants use their working memory allows us to analyze the perceptual qualities of the secondary information in the AR headset. In our study, participants completed single-digit $\mathrm{x}$ three-digit multiplication problems. We chose multiplication because it takes more time and attention to solve than addition [20]. None of the participants saw the same problem twice. In the application, the current problem would appear at the top and participants could work out the problem with a stylus pen (Figure 1). The participants had to enter an answer, which did not have to be correct, before hitting "next". There was not a set amount of problems; each participant completed the amount they could do in the block time frame. Participants were instructed to take their time and focus on getting the correct answers.

\section{Data Analysis and Results}

We analyzed the different presentation styles by examining the participants' math solve time, cognitive workload, and accuracy of recalled information. After each study block, participants were asked to recall the last textual information that was presented in the AR headset. Since we did not inform the participants that we would ask them to recall the information, the participants were unaware for the first block; however, after the first survey the participants became aware. Therefore, we split the analysis for recalled information into: unaware recall (first style) and aware recall (other styles). Unaware recall captures the raw perceptibility of the presentation styles, while aware recall coincides with real-world settings in which the users are aware of the task. A Shapiro-Wilks test showed that the data was nonnormal for all metrics. Therefore, we applied the Aligned Rank Transform [31] to each metric. If significant, a Tukey post-hoc comparison was applied (unless stated otherwise).

For unaware recall, we calculated the proportion of correct answers for each participant's first presentation style. An answer 
was considered correct if it exactly matched the last information presented in the headset. An ANOVA found no significant effect of type of presentation style on unaware recall accuracy $\left(\mathrm{F}_{2,27}=\right.$ 1.64, n.s.). When the participants did not know they had to recall the information, there was no significant difference between the Display $(M=60 \%, S D=21.1 \%)$, Environment $(M=67.5 \%, S D=$ $23.7 \%)$, and Mixed Environment $(M=50 \%, S D=20.4 \%)$ styles. We analyzed aware recall the same way as unaware recall, but for each participant's second and third presentation styles. A RMANOVA found a significant main effect of type of presentation type on aware recall accuracy $\left(\mathrm{F}_{2,44}=8.91, \mathrm{p}<0.0001\right)$. Participants had a significantly higher aware recall accuracy for Environment $(M=83.8 \%, S D=18.6 \%)$ than Mixed Environment $(\mathrm{M}=51.2 \%, \mathrm{SD}=30.9 \%)$. We did not find a significant difference between Environment and Display $(\mathrm{M}=68.8 \%, \mathrm{SD}=21.3 \%)$. Since aware recall was significant, we examined each secondary information separately (average math time, math accuracy, random word). We only found a significant effect of presentation style on the random word accuracy ( $p<0.001$, Fisher's exact test [13]). A pairwise test of independence with a Bonferroni correction only found a significant difference between the Environment and Mixed Environment styles. Environment had a higher count of correct random word recall events (18 correct, 2 incorrect) than Mixed Environment (6 correct, 14 incorrect).

Also, we examined the math solve time (i.e., time from when the current problem appeared to when the next button was hit), in order to see if participants were consistently focusing on the math application. Since we instructed participants to take their time, we did not analyze solve time as an individual metric (i.e., we were not interested in speed). Rather we use solve time as a proxy for determining if there was a shift in focus between the $\mathrm{AR}$ and math application for the types of styles. A RM-ANOVA found no significant effect of presentation style on solve time $\left(\mathrm{F}_{2,58}=0.77\right.$, n.s. $)$. The participants' consistency in solve time reinforces that participants focused on the math problems as their main task. Lastly, we analyzed the participants' perceived cognitive workload for each presentation style. A RM-ANOVA found no significant effect of type of presentation style on perceived cognitive workload $\left(\mathrm{F}_{2,58}=0.12\right.$, n.s. $)$.

\section{Discussion}

Overall, we did not find a main significant difference between the three presentation styles for textual secondary information. However, we did find a significantly higher aware recall accuracy for Environment compared to Mixed Environment. When further examining aware recall, we only found a significant effect of presentation style on the random word accuracy. Participants frequently remarked that they were more interested in the other textual information (e.g., math accuracy) since it directly pertained to the main task. Therefore, the Environment style aided in the awareness of non-pertinent information, which highlights the high perceptibility of the style.

For the Mixed Environment style, having the text $500 \mathrm{~mm}$ away and always present put the information more in the participants' field of vision, which made it more distracting to the participants. For example, P13 stated "The mixed environment was too distracting and put too much pressure on me to get more problems right". With the Environment presentation style, the participants could look at the information when they desired. P8 stated "[The Environment style] didn't get in my way so I didn't have to block it out of my vision while completing the math problems. It was nice to look up at it when I felt the need to." Although the Display style was always present in the field-ofview, participants remarked that it was easier to tune out since it was located more in the periphery. Both the Display and Environment presentation styles allowed the participants to tune out and view the secondary information when they preferred, resulting in a stronger focus and higher recall accuracy.

In our study, both the Display and Environment styles improved perception and comprehension for secondary textual information. However, prior work in virtual reality has found that text notifications locked to the display result in a higher sense of urgency than text notifications floating in the environment [26]. Secondary information should be unobtrusive and not require a sense of urgency [8]. Therefore, we recommend that designers utilize the Environment style for nonessential secondary information and the Display style for more important information in AR headsets.

\section{Limitations and Future Work}

Our work supports the design of secondary information in AR headsets for situational awareness. Still, there are some limitations to the scope of our work. First, we only focused on perception (level 1) and comprehension (level 2) for three presentation styles. In addition, the environment and amount of information could have affected perceptibility. We view our study as a starting point for examining how to design information in AR headsets for situational awareness. Future work can investigate other types of information in different environments, as well as focus more on prediction (level 3).

\section{Conclusion}

We conducted a study on how to present textual secondary information in augmented reality (AR) headsets for situational awareness (perception and comprehension). We examined three different presentation styles: Display, Environment, and Mixed Environment. Our analysis revealed that the Display and Environment presentation styles improved perception and comprehension for secondary information; participants had a higher recall of information when compared to the Mixed Environment style. Our work is a first step in designing information in AR headsets to increase situational awareness.

\section{ACKNOWLEDGMENTS}

This work is partially supported by National Science Foundation Grant Award \#IIS-1750840 and the National Science Foundation Graduate Research Fellowship under Grant No. DGE-1842473. Any opinions, findings, and conclusions or recommendations expressed in this paper are those of the authors and do not necessarily reflect these agencies' views. 


\section{REFERENCES}

[1] Andrea Albarelli, Augusto Celentano, Luca Cosmo, and Renato Marchi. 2015. On the Interplay between Data Overlay and Real-World Context using Seethrough Displays. In Proceedings of the 11th Biannual Conference on Italian SIGCHI Chapter (CHItaly'15), DOI:https://doi.org/10.1145/2808435.2808455

[2] Doris Aschenbrenner, Nicolas Maltry, Johannes Kimmel, Michael Albert, Julian Scharnagl, and Klaus Schilling. 2016. ARTab - Using Virtual and Augmented Reality Methods for an Improved Situation Awareness for Telemaintenance. IFAC-PapersOnLine 49, 30 (2016), 204-209. DOI:https://doi.org/10.1016/J.IFACOL.2016.11.168

[3] Alan Baddeley. 1992. Working Memory. Science 255, 556-559. DOI:https://doi.org/10.1126/SCIENCE.1736359

[4] Mon-Chu Chen and Roberta Klatzky. 2007. Displays Attentive to Unattended Regions: Presenting Information in a Peripheral-Vision-Friendly Way. Int. Conf. Human-Computer Interact. 4551, (2007), 23-31.

[5] Saverio Debernardis, Michele Fiorentino, Michele Gattullo, Giuseppe Monno, and Antonio Emmanuele Uva. 2014. Text Readability in Head-Worn Displays: Color and Style Optimization in Video versus Optical See-Through Devices. IEEE Trans. Vis. Comput. Graph. 20, 1 (January 2014), 125-139. DOI:https://doi.org/10.1109/TVCG.2013.86

[6] Mica R. Endsley. 1988. Design and Evaluation for Situation Awareness Enhancement. Proc. Hum. Factors Soc. Annu. Meet. 32, 2 (1988), 97-101. DOI:https://doi.org/10.1177/154193128803200221

[7] Mica R. Endsley. 1995. Toward a Theory of Situation Awareness in Dynamic Systems. Hum. Factors 7. Hum. Factors Ergon. Soc. 37, 1 (1995), 32-64 DOI:https://doi.org/10.1518/001872095779049543

[8] Mica R. Endsley. 2001. Designing for Situation Awareness in Complex System. In Proceedings of the Second International Workshop on Symbiosis of Humans, Artifacts and Environment, 14pp.

[9] Subhashini Ganapathy. 2013. Design Guidelines for Mobile Augmented Reality: User Experience. In Human Factors in Augmented Reality Environments. Springer New York, New York, NY, 165-180. DOI:https://doi.org/10.1007/978-1-4614-4205-9_7

[10] Eric Gans, David Roberts, Matthew Bennett, Herman Towles, Alberico Menozzi, James Cook, and Todd Sherrill. 2015. Augmented Reality Technology for Day/Night Situational Awareness for the Dismounted Soldier. In Display Technologies and Applications for Defense, Security, and Avionics, Article 9470. DOI:https://doi.org/10.1117/12.2177086

[11] Sandra G. Hart. 2006. Nasa-Task Load Index (NASA-TLX); 20 Years Later. Proc. Hum. Factors Ergon. Soc. Annu. Meet. 50, 9 (October 2006), 904-908. DOI:https://doi.org/10.1177/154193120605000909

[12] Yoshio Ishiguro and Jun Rekimoto. 2011. Peripheral Vision Annotation: Noninterference Information Presentation Method for Mobile Augmented Reality. In Proceedings of the Augmented Human International Conference (AH'11), 1-5. DOI:https://doi.org/10.1145/1959826.1959834

[13] Hae-Young Kim. 2017. Statistical Notes for Clinical Researchers: Chi-squared Test and Fisher's Exact Test. Restor. Dent. Endod. 42, 2 (May 2017), 152-155. DOI:https://doi.org/10.5395/rde.2017.42.2.152

[14] David Liu, Simon A. Jenkins, Penelope M. Sanderson, Marcus O. Watson, Terrence Leane, Amanda Kruys, and W John Russell. 2009. Monitoring with Head-Mounted Displays: Performance and Safety in a Full-Scale Simulator and Part-Task Trainer. Anesth. Analg. 109, 4 (October 2009), 1135-1146. DOI:https://doi.org/10.1213/ANE.0b013e3181b5a200

[15] Robert H. Logie, Kenneth J. Gilhooly, and Valerie Wynn. 1994. Counting on Working Memory in Arithmetic Problem Solving. Mem. Cognit. 22, 4 (July 1994), 395-410. DOI:https://doi.org/10.3758/BF03200866

[16] Stephan Lukosch, Heide Lukosch, Dragoş Datcu, and Marina Cidota. 2015. Providing Information on the Spot: Using Augmented Reality for Situational Awareness in the Security Domain. Comput. Support. Coop. Work 24, 6 (December 2015), 613-664. DOI:https://doi.org/10.1007/s10606-015-9235-4

[17] Aristides Mairena, Carl Gutwin, and Andy Cockburn. 2019. Peripheral Notifications in Large Displays: Effects of Feature Combination and Task Interference. In Proceedings of the SIGCHI Conference on Human Factors in Computing Systems (CHI'19), 12pp. DOI:https://doi.org/10.1145/3290605.3300870

[18] Meta. Meta Augmented Reality. Retrieved April 24, 2019 from https://www.metavision.com/

[19] Meta. Meta Interface Design. Retrieved April 24, 2019 from https://www.metavision.com/interface-design/

[20] Kevin Miller, Marion Perlmutter, and Daniel Keating. 1984. Cognitive Arithmetic: Comparison of Operations. 7. Exp. Psychol. Learn. Mem. Cogn. 10, 1 (1984), 46-60. DOI:https://doi.org/10.1037/0278-7393.10.1.46

[21] Jason Orlosky, Kiyoshi Kiyokawa, and Haruo Takemura. 2014. Managing Mobile Text in Head Mounted Displays: Studies on Visual Preference and Text Placement. ACM SIGMOBILE Mob. Comput. Commun. Rev. 18, 2 (June 2014), 20-31. DOI:https://doi.org/10.1145/2636242.2636246

[22] Byoung-Jun Park, Changrak Yoon, Jeong-Woo Lee, and Kyong-Ho Kim. 2015.
Augmented Reality Based on Driving Situation Awareness in Vehicle. In International Conference on Advanced Communication Technology (ICACT'15), 593-595. DOI:https://doi.org/10.1109/ICACT.2015.7224865

[23] Michael T. Pascale, Penelope Sanderson, David Liu, Ismail Mohamed, Birgit Brecknell, and Robert G. Loeb. 2019. The Impact of Head-Worn Displays on Strategic Alarm Management and Situation Awareness. Hum. Factors 61, 4 (2019), 537-563. DOI:https://doi.org/10.1177/0018720818814969

[24] Luz Rello and Ricardo Baeza-Yates. 2017. How to Present More Readable Text for People with Dyslexia. Univers. Access Inf. Soc. 16, 1 (March 2017), 29-49. DOI:https://doi.org/10.1007/s10209-015-0438-8

[25] Susana Ruano, Carlos Cuevas, Guillermo Gallego, and Narciso García. 2017 Augmented Reality Tool for the Situational Awareness Improvement of UAV Operators. $\quad$ Sensors 17, 2 (February 2017), 297. DOI:https://doi.org/10.3390/s17020297

[26] Rufat Rzayev, Sven Mayer, Christian Krauter, and Niels Henze. 2019. Notification in VR: The Effect of Notification Placement, Task, and Environment. In Computer-Human Interaction in Play (CHIPlay'19), 199-211. DOI:https://doi.org/10.1145/3311350.3347190

[27] Rufat Rzayev, Paweł W. Wozniak, Tilman Dingler, and Niels Henze. 2018 Reading on Smart Glasses: The Effect of Text Position, Presentation Type and Walking. In Proceedings of the SIGCHI Conference on Human Factors in Computing Systems (CHI'18), $\quad 9 \quad$ pages. DOI:https://doi.org/10.1145/3173574.3173619

[28] Christian M Schulz, Veronika Krautheim, Annika Hackemann, Matthias Kreuzer, Eberhard F Kochs, and Klaus J Wagner. 2016. Situation Awareness Errors in Anesthesia and Critical care in 200 Cases of a Critical Incident Reporting System. BMC Anesthesiol. 16, $4 \quad$ (2016) DOI:https://doi.org/10.1186/s12871-016-0172-7

[29] Neville A. Stanton, Peter R.G. Chambers, and John Piggott. 2001. Situational Awareness and Safety. Saf. Sci. 39, 3 (December 2001), 189-204. DOI:https://doi.org/10.1016/S0925-7535(01)00010-8

[30] Wacom. Professional Hybrid Creative Tablet User's Manual About the Cintiq Companion Hybrid. 1-93. Retrieved from http://support.wacom.com.hk/manuals

[31] Jacob O. Wobbrock, Leah Findlater, Darren Gergle, and James J. Higgins. 2011. The Aligned Rank Transform for Nonparametric Factorial Analyses Using Only Anova Procedures. In Proceedings of the SIGCHI Conference on Human Factors in Computing Systems (CHI'11), 143-146. DOI:https://doi.org/10.1145/1978942.1978963

[32] Zhiwei Zhu, Vlad Branzoi, Michael Wolverton, Glen Murray, Nicholas Vitovitch, Louise Yarnall, Girish Acharya, Supun Samarasekera, and Rakesh Kumar. 2014. AR-Mentor: Augmented Reality Based Mentoring System. In IEEE International Symposium on Mixed and Augmented Reality (ISMAR), 1722. DOI:https://doi.org/10.1109/ISMAR.2014.6948404

[33] Stefanie Zollmann, Christof Hoppe, Tobias Langlotz, and Gerhard Reitmayr 2014. FlyAR: Augmented Reality Supported Micro Aerial Vehicle Navigation. IEEE Trans. Vis. Comput. Graph. 20, 4 (April 2014), 560-568. DOI:https://doi.org/10.1109/TVCG.2014.24

[34] Augmented Reality Is A Game Changer For Oil \& Gas. OilPrice.com. Retrieved from https://oilprice.com/Energy/Energy-General/Augmented-Reality-Is-AGame-Changer-For-Oil-Gas.html 